\title{
Left main disease diagnosis: The Achilles heel or the great strength of modern cardiac imaging?
}

\author{
Aiden Abidov, MD, PHD, FACC, FAHA ${ }^{\mathrm{a}}$ \\ a Section of Cardiology, Department of Medicine, The University of Arizona, Tucson, AZ \\ Received Sep 29, 2015; accepted Sep 29, 2015 \\ doi:10.1007/s12350-015-0306-1
}

\section{See related article, pp. 1411-1429}

In this issue of the Journal, Sareen and Ananthasubramaniam provide an extensive review of the available evidence describing the value of rest and stress electrocardiography, nuclear myocardial perfusion imaging, stress echocardiography, cardiac computed tomography, and cardiac magnetic resonance imaging in diagnosis of left main (LM) coronary artery disease (CAD). ${ }^{1}$ The authors included a total of 102 references covering this important topic and provided several very interesting Figures. I would specifically mention their Figure 9, with the proposed algorithm and suggestions for utilization of multiple diagnostic imaging modalities as well as Table 1, with a summary of diagnostic strengths and weaknesses for the noninvasive diagnostic modalities reviewed in this paper.

Any practicing cardiologist would have agreed with the authors in their conclusions. Based on their analysis, the authors state that " "...noninvasive detection of significant LM stenosis is critically important given the underlying extent of myocardium at risk'. ${ }^{1}$ They also rightfully conclude that all the reviewed noninvasive methodologies have their intrinsic limitations in assessment of extent of LM disease.

Among the methodologies reviewed and compared in this paper, cardiac CTA (CCTA) appears to be the most promising test for evaluation of LM stenosis. The strength of physiologic testing, including nuclear perfusion imaging, appears to be good, but not perfect. Evidence reviewed by the authors suggests a value of

Reprint requests: Aiden Abidov, MD, PHD, FACC, FAHA, Section of Cardiology, Department of Medicine, The University of Arizona,

Tucson, AZ; aabidov@shc.arizona.edu

J Nucl Cardiol 2016;23:1430-4.

$1071-3581 / \$ 34.00$

Copyright (C) 2015 American Society of Nuclear Cardiology. the integrative approach. Taking into consideration the hemodynamic and ECG response to stress on top of the imaging findings may definitely help suspect the presence of LM stenosis or extensive multivessel disease. Finally, the authors suggest that when the noninvasive tests are equivocal, clinical suspicion should prevail, warranting invasive coronary angiography.

This paper of Sareen and Ananthasubramaniam raises several important clinical concerns. Currently, the option of choosing medical therapy vs intervention is frequently discussed with patients based on the COURAGE trial results. ${ }^{2}$ However, it is important to note that all patients with either high-risk scans or highrisk anatomy (including 947 patients with LM disease) were excluded from the COURAGE study population. In this regard, the COURAGE approach applies only to patients with a stable non-high-risk CAD. An important conclusion here is that clinicians must perform either anatomic or physiologic testing before offering a medical treatment option to patients with CAD.

When appropriately diagnosed, surgical treatment of LM disease may substantially improve morbidity and mortality, ${ }^{3}$ while misdiagnosis of this serious disorder may lead to an extensive myocardial infarction (MI) or death. ${ }^{4}$ Unfortunately, a significant percent of these patients may have either fatal MI or sudden death as their initial presentation.

Even when patients with angiographically significant (including $>50 \%$ stenosis) LM disease have normal stress MPI results, their death or MI rate reaches $2 \% /$ year, despite the pervasive belief that normal stress imaging is associated with benign prognosis. ${ }^{5}$

As one can see from the available literature review, we are not yet perfect in our ability to diagnose LM disease based on use of any diagnostic imaging modality. Berman et al demonstrated that based on myocardial perfusion results alone (presence of $>10 \%$ of the myocardium ischemic), LM disease may be under-diagnosed in more than $40 \%$ of cases. ${ }^{6}$ A really sobering fact in this paper was that up to $15 \%$ of the LM disease cases did 
not have any perfusion abnormality. Thus, LM disease may represent an "Achilles heel" of the diagnostic imaging.

Physiologic testing may miss/underestimate the presence of the LM disease due to a variety of reasons. Among the frequent factors limiting diagnostic performance are imaging artifacts, gating errors, quality issues, as well as inability of the patient to perform a complete exercise stress MPI test (defined as achieving the maximal predicted heart rate AND ability to walk on a treadmill for at least $6 \mathrm{~min}$ ). In addition, the wellknown phenomenon of balanced ischemia (resulting in an apparently normal-appearing perfusion map) was described for stress MPI applications decades ago.

The "classic appearance" of the high-risk MPI scan in patients with LM disease reveals severe perfusion defects in the LAD and LCX territories (Figure 1). However, some MPI studies in patients with LM disease demonstrate perfusion abnormalities in only one of the vascular territories (either LAD or LCX) (Figure 2).

In patients with LM disease, both stress-induced ECG changes and hemodynamic response (hypotensive response to exercise), as well as stress-induced ischemic arrhythmias (especially polymorphic VT), may help raise suspicion of severe $\mathrm{CAD} / \mathrm{LM}$ disease. However, ECG in general has only a modest diagnostic value in predicting LM disease. As an example, one of our patients with severe distal LM and subtotal mid-LAD disease (Figure 3) was admitted recently with unstable angina; her serial $(\times 3)$ cardiac troponins and ECGs remained normal. The patient was considered to be at high risk and referred for coronary angiogram solely based on her clinical presentation.

Ancillary non-perfusion markers of severe ischemia (transient ischemic dilation-TID; abnormal TID ratio; increased lung heart ratio; or increased lung uptake,

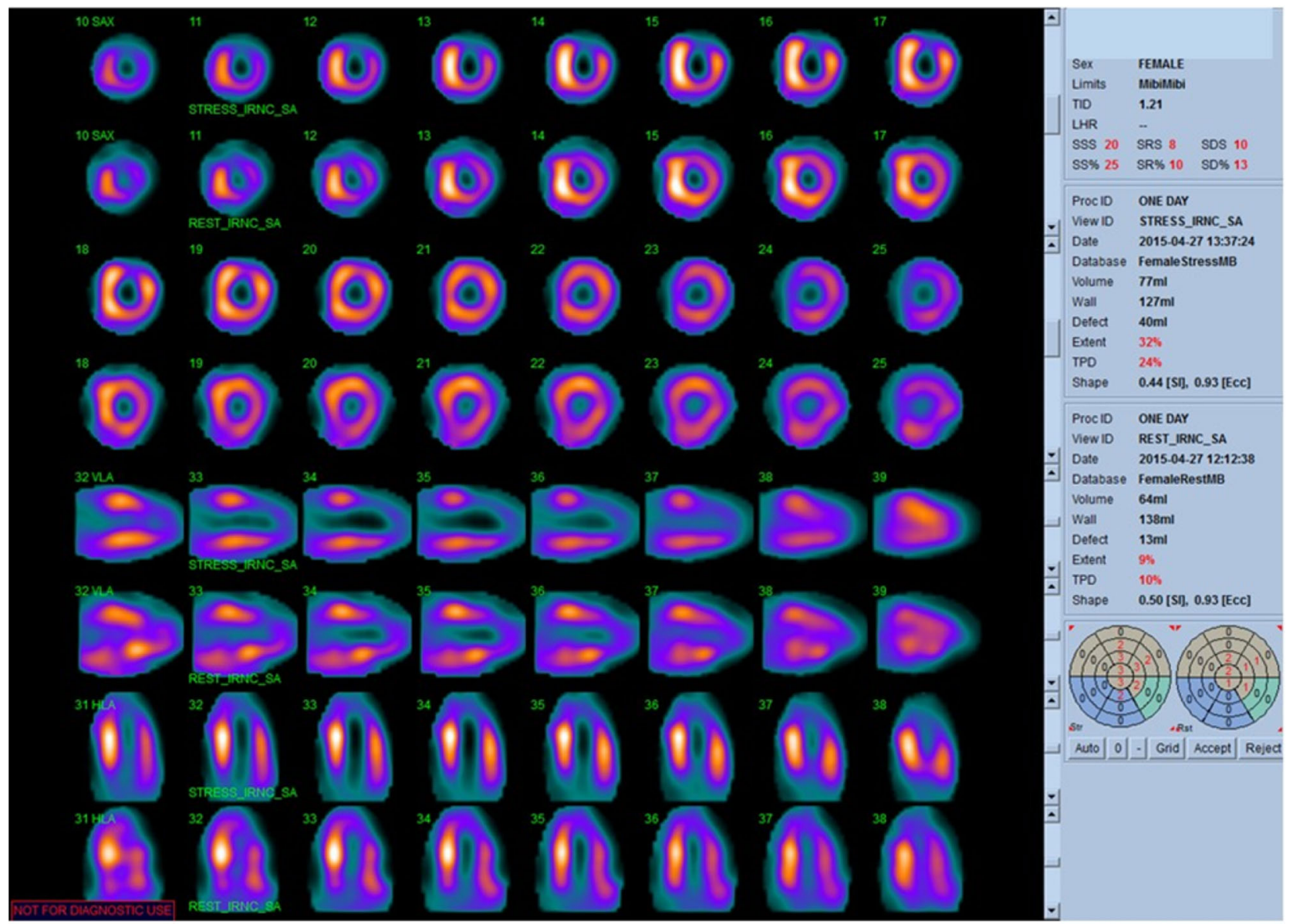

Figure 1. Exercise stress Tc-99m Sestamibi single day myocardial perfusion SPECT images of the female patient with a significant $(80 \%)$ distal left main coronary artery disease. Classic features of the high-risk scan are present: severe partially reversible perfusion defect, involvement of the LAD and LCX territory, visual transient ischemic dilation and abnormal TID ratio (1.21). The patient presented with symptoms of stable atypical angina. No significant ECG or hemodynamic changes were noted during the stress portion of the test. 

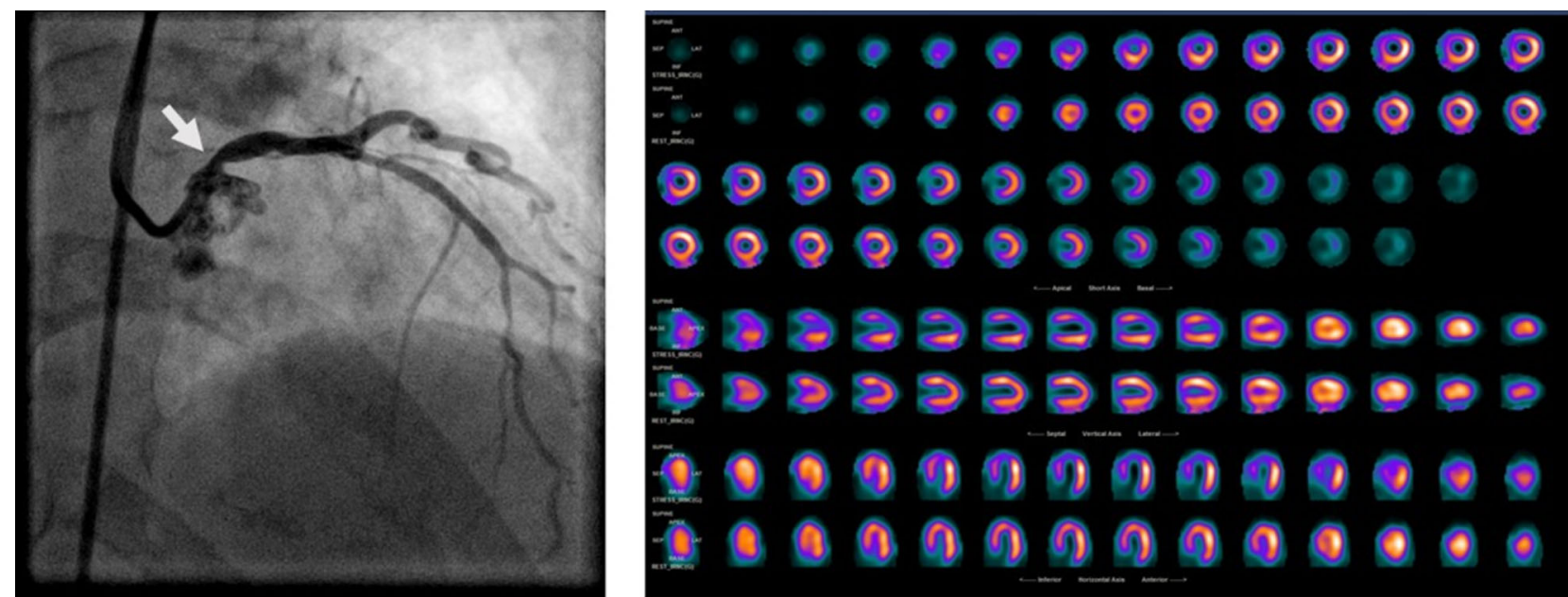

Figure 2. Evidence of a significant (80\% stenosis) proximal left main disease (white arrow) in a female patient with stable angina. No significant LAD disease is present. Stress myocardial perfusion images revealed severe reversible perfusion defect in the LAD territory; no ischemia is seen in the LCX territory. Abnormal visual TID and TID ratio suggests the presence of the severe and extensive CAD.

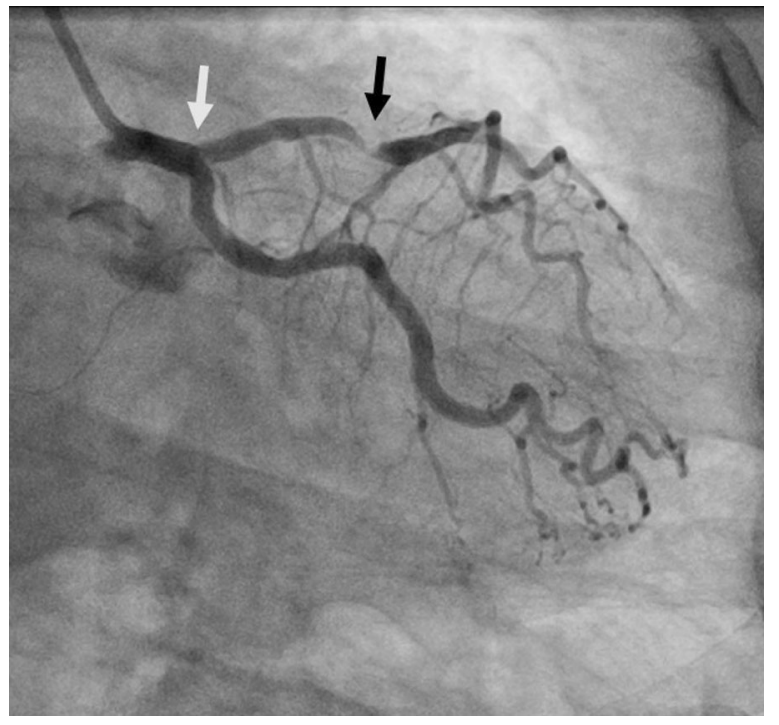

Figure 3. Evidence of a significant (70\%) distal left main/ ostial LAD (white arrow) and additional subtotal mid-LAD stenosis (black arrow) in a female patient with typical recent onset (3 days) unstable (progressive) exertional angina, normal resting ECG, and normal serial troponins.

decrease in ejection fraction with stress-ischemic stunning; focal left ventricular geometry change; etc.) have shown a good predictive value in distinguishing patients with high risk and severe and extensive CAD. ${ }^{7-15}$ In two examples of patients with LM disease (Figures 1, 2), TID ratio and visual TID were abnormal. Use of these markers requires careful assessment of the scan quality, is tracer, stressor, and protocol-dependent, ${ }^{8}$ and has to take into consideration the presence of perfusion abnormalities ${ }^{16}$ and the clinical likelihood of CAD. ${ }^{17}$

In general, a combination of clinical, hemodynamic, electrocardiographic, and perfusion imaging parameters with ancillary markers may increase the diagnostic power of stress MPI in predicting LM disease, ${ }^{6}$ as well as the overall diagnostic certainty of the imaging test. ${ }^{18}$ This combined diagnostic power is more accurate in predicting LM disease than any other clinical methodology, and should be considered a true strength of the modern cardiac imaging.

Unlike physiologic imaging, anatomic imaging (either coronary CTA or invasive coronary angiogram) directly visualizes coronary stenosis and obstruction (Figure 4). One well-known application of the CTA is its utilization in patients with equivocal or nondiagnostic stress MPI results. ${ }^{19}$ The potential of CTA is very promising in patients with significant ischemia who are candidates for PCI, as the CTA can predict success of PCI based on the plaque characteristics, ${ }^{20}$ and also potentially help in the revascularization (surgical vs PCI) planning. CTA also prevents starting dual antiplatelet therapy in patients who have a severe LM/3 vessel CAD and may need urgent surgical revascularization.

Modern anatomic imaging may also prove helpful in patients with known CAD and history of prior LM intervention (PCI to LM). CCTA in these cases may demonstrate not only LM in-stent restenosis but also reveal progression of coronary atherosclerosis in the non-LM locations and define need and time for revascularization. The potential of CTA perfusion, and 

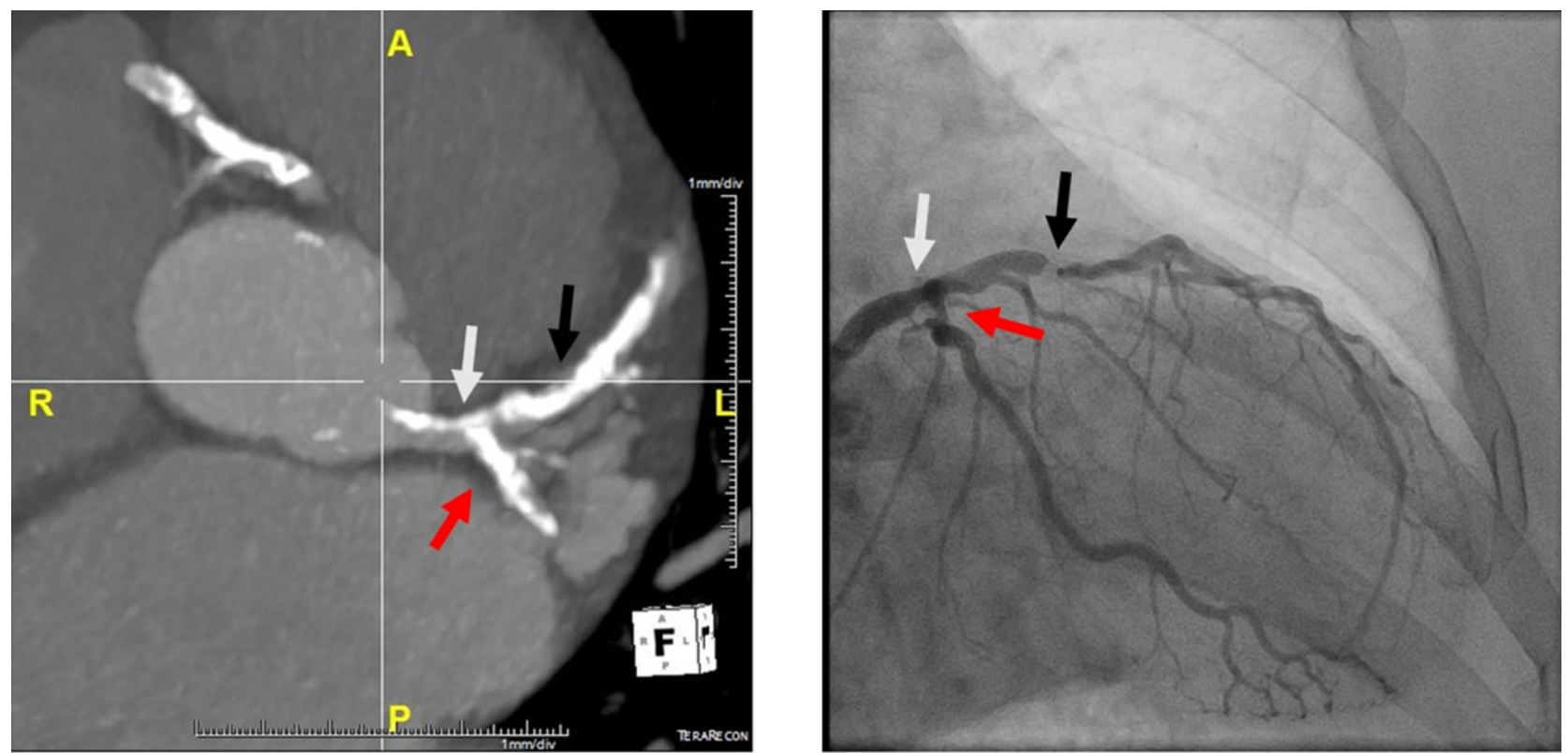

Figure 4. Incidental finding of severe calcified distal left main and ostial LAD (white arrow), midLAD (black arrow) and LCX disease (red arrow) on chest CTA performed to evaluate a thoracic aorta in an asymptomatic elderly male patient with a history of TIA. Cardiac catheterization confirmed the CTA results.

especially CT-FFR for diagnosis of LM disease, still needs to be defined in further research.

Coronary CTA may have the capability to predict future adverse events even when a nonobstructive $(<50 \%)$ coronary plaque is found in the LM. Compared to invasive coronary angiogram with visualization of the "lumenogram," the expectation for prediction of the plaque behavior based on the CTA plaque vulnerability indicators is much higher.

Based on evidence published in the last decade, the problem of "Achilles heel" of the cardiac imaging may definitively be solved using an integrative approach to scan interpretation. Especially important here are recent publications of Slomka et $\mathrm{al}^{21-23}$ comparing artificial intelligence (AI) program learning vs expert interpretation of the myocardial perfusion scans; in these papers, the AI performance was excellent and appeared similar or better when compared to the expert reads. While a methodology of integrated reads with global analysis of the entire dataset has been described, ${ }^{18}$ only AI-based reading algorithms will allow analysis of all the complex data available from the noninvasive imaging modality, add to this analysis all clinical variables and any ancillary findings, and come up with a diagnosis specifically developed for a particular patient. AI-based assistance is invaluable for all the versatile imaging modalities, including nuclear myocardial perfusion imaging (SPECT or cardiac PET), Cardiac CCTA (adding together the vessel lumen and wall/plaque structure information, perfusion, flow and global and regional function), and Cardiac MRI. Current electronic image fusion may lead to future data analysis algorithms from several imaging modalities, with development of individualized $\mathrm{LM}$ /significant CAD probability models.

In summary, based on modern diagnostic tools (especially CTA), cardiac imaging allows us to have a much better understanding of LM disease, including the precise location, severity, extent and physiologic significance of the lesion. Moreover, based on plaque characteristics, we can predict future risk associated not only with obstructive, but also with nonobstructive LM disease. It seems logical to combine physiologic and anatomic imaging results for the best diagnostic effectiveness in patients with known or suspected LM disease. Future AI applications with automated analysis of the entire imaging and nonimaging dataset from a single or multiple tests may further enhance our ability to create individualized diagnostic and prognostic models for patients and provide a choice of the best possible management based on these models.

I believe we are already reaching the time point where diagnosis of the high risk CAD, and especially LM disease turns into a great strength of modern cardiac imaging.

How many saved lives will our enhanced diagnostic power lead to-is yet to be assessed in future research. 


\section{References}

1. Sareen N, Ananthasubramaniam K. Left main coronary artery disease: A review of the spectrum of non-invasive diagnostic modalities. J Nucl Cardiol 2015;65:A122.

2. Boden WE, O'Rourke RA, Teo KK, Hartigan PM, Maron DJ, Kostuk W, et al. The evolving pattern of symptomatic coronary artery disease in the United States and Canada: Baseline characteristics of the Clinical Outcomes Utilizing Revascularization and Aggressive Drug Evaluation (COURAGE) trial. Am J Cardiol 2007;99:208-12.

3. Campeau L, Corbara F, Crochet D, Petitclerc R. Left main coronary artery stenosis: The influence of aortocoronary bypass surgery on survival. Circulation 1978;57:1111-5.

4. Cohen MV, Gorlin R. Main left coronary artery disease. Clinical experience from 1964-1974. Circulation 1975;52:275-85.

5. Agarwal V, Yao SS, Chaudhry FA. Utilization of stress echocardiography in patients with multivessel coronary artery disease. $\mathrm{J}$ Cardiovasc Med (Hagerstown) 2015;22:e77.

6. Berman DS, Kang X, Slomka PJ, Gerlach J, de Yang L, Hayes SW, et al. Underestimation of extent of ischemia by gated SPECT myocardial perfusion imaging in patients with left main coronary artery disease. J Nucl Cardiol 2007;14:521-8.

7. Mazzanti M, Germano G, Kiat H, Kavanagh PB, Alexanderson E, Friedman JD, et al. Identification of severe and extensive coronary artery disease by automatic measurement of transient ischemic dilation of the left ventricle in dual-isotope myocardial perfusion SPECT. J Am Coll Cardiol 1996;27:1612-20.

8. Abidov A, Germano G, Berman DS. Transient ischemic dilation ratio: A universal high-risk diagnostic marker in myocardial perfusion imaging. J Nucl Cardiol 2007;14:497-500.

9. Ilmer B, Reijs AE, Reiber JH, Bakker W, Fioretti P. Relationships between the lung-heart ratio assessed from post-exercise thallium201 myocardial tomograms, myocardial ischemia and the extent of coronary artery disease. Int J Card Imaging 1990;6:135-41.

10. Castellani M, Chiti A, Giovanella LC, Bestetti A, Lomuscio A, Tarolo GL. Thallium-201 lung uptake: Comparison of an automatic and a manual method of ROI drawing. J Nucl Biol Med 1993;37:213-7.

11. Germano G, Kavanagh PB, Berman DS. An automatic approach to the analysis, quantitation and review of perfusion and function from myocardial perfusion SPECT images. Int J Card Imaging 1997; 13:337-46.

12. Narula J, Dawson MS, Singh BK, Amanullah A, Acio ER, Chaudhry FA, et al. Noninvasive characterization of stunned, hibernating, remodeled and nonviable myocardium in ischemic cardiomyopathy. J Am Coll Cardiol 2000;36:1913-9.
13. Schinkel AF, Bax JJ, van Domburg R, Elhendy A, Valkema R, Vourvouri EC, et al. Dobutamine-induced contractile reserve in stunned, hibernating, and scarred myocardium in patients with ischemic cardiomyopathy. J Nucl Med 2003;44:127-33.

14. Ben-Haim S, Gips S, Merdler A, Front A, Tamir A. Myocardial stunning demonstrated with rest and post-stress measurements of left ventricular function using dual-isotope gated myocardial perfusion SPECT. Nucl Med Commun 2004;25:657-63.

15. Druz RS, Akinboboye OA, Grimson R, Nichols KJ, Reichek N. Postischemic stunning after adenosine vasodilator stress. J Nucl Cardiol 2004;11:534-41.

16. Abidov A, Bax JJ, Hayes SW, Cohen I, Nishina H, Yoda S, et al. Integration of automatically measured transient ischemic dilation ratio into interpretation of adenosine stress myocardial perfusion SPECT for detection of severe and extensive CAD. J Nucl Med 2004;45:1999-2007.

17. Abidov A, Bax JJ, Hayes SW, Hachamovitch R, Cohen I, Gerlach $\mathrm{J}$, et al. Transient ischemic dilation ratio of the left ventricle is a significant predictor of future cardiac events in patients with otherwise normal myocardial perfusion SPECT. J Am Coll Cardiol 2003;42:1818-25.

18. Abidov A, Hachamovitch R, Hayes SW, Friedman JD, Cohen I, Kang X, et al. Are shades of gray prognostically useful in reporting myocardial perfusion single-photon emission computed tomography? Circ Cardiovasc Imaging 2009;2:290-8.

19. Abidov A, Gallagher MJ, Chinnaiyan KM, Mehta LS, Wegner JH, Raff GL. Clinical effectiveness of coronary computed tomographic angiography in the triage of patients to cardiac catheterization and revascularization after inconclusive stress testing: Results of a 2year prospective trial. J Nucl Cardiol 2009;16:701-13.

20. Fujimoto S, Kondo T, Kumamaru KK, Shinozaki T, Takamura K, Kawaguchi Y, et al. Prognostic value of coronary computed tomography (CT) angiography and coronary artery calcium score performed before revascularization. J Am Heart Assoc 2015;4: e002264.

21. Arsanjani R, Dey D, Khachatryan T, Shalev A, Hayes SW, Fish M, et al. Prediction of revascularization after myocardial perfusion SPECT by machine learning in a large population. J Nucl Cardiol 2015;22:877-84.

22. Kang D, Slomka PJ, Nakazato R, Arsanjani R, Cheng VY, Min JK, et al. Automated knowledge-based detection of non obstructive and obstructive arterial lesions from coronary CT angiography. Med Phys 2013;40:041912.

23. Arsanjani R, Xu Y, Hayes SW, Fish M, Lemley M Jr, Gerlach J, et al. Comparison of fully automated computer analysis and visual scoring for detection of coronary artery disease from myocardial perfusion SPECT in a large population. J Nucl Med 2013;54:221-8. 